\title{
Particle Swarm Optimization Based Discrete Cosine Transform for Person Identification by Gait Recognition
}

\author{
Shahlla A. AbdAlKader \\ Dept. of Computer Systems, Foundation of Technical Education \\ Technical Institute, Mosul, Iraq \\ shahla_ak71@yahoo.com \\ Omaima N. Ahmad AL-Allaf \\ Dept. of Basic Sciences, \\ Faculty of Sciences and Information Technology \\ AL-Zaytoonah University of Jordan, P.O. Box 130, Amman (11733), Jordan \\ omaimaalallaf@zuj.edu.jo
}

\begin{abstract}
Gait recognition addresses the problem of human identification at a distance by identifying people based on the way they walk. Therefore, gait recognition has gained growing interest from researchers in recent years. This work presents gait recognition system based on particle swarm optimization (PSO) to recognize a person performing the movement for person identification. The system is based on Discrete Cosine Transform (DCT) for reducing dimensionality and feature extraction. Many experiments were conducted using different: swarm size, block dimension and number of iterations. The results showed that increasing the swarm size to 40 particles and also increasing block size of DCT sub image to $(\mathbf{7 0 \times 7 0})$ pixels will increase the overall performance of gait recognition system. The recognition rate reached $96 \%$, MSE reached 0.0088 and PSNR reached $35 \%$.
\end{abstract}

Keywords- Gait Recognition, Person Identification, Practical Swarm Optimization (PSO), Discrete Cosine Transform (DCT)

\section{INTRODUCTION}

Person identification can associate an identity to any person. Recently, person identification is highly researched according to its applications such as: authentication to computer systems, buildings, cellular phones and ATMs [1].

Person identification includes many techniques such as tokenbased, knowledge-based, and biometric-based. Knowledgebased technique depends on something a person knows for identification like password or personal identification number (PIN). Token-based technique depends on something a person has for identification (passport, driver's license, ID card, keys, or credit card). The disadvantages of the two approaches are: tokens may be stolen, lost, forgotten or misplaced. The biometric technique uses physiological or behavioral features of person for identification and it cannot be lost [2]. Fingerprint recognition, iris recognition, face recognition, speech recognition are some of biometric-based techniques [3]. For person identification, these techniques require controlled environment and the person should stand at a standard distance in front of a camera. Therefore, these techniques cannot be used in automatic surveillance of people in real time situations. For this reason, gait recognition has been widely used to provide noninvasive way to recognize persons at a distance without requiring the awareness of the identified person. Many researches based on gait recognition methods have been proposed in the last decade [1].

Gait or motion can be defined as a sequence of the following poses that recognize people as well as walking. Kinematic chain is a typical representation of a single pose. It describes the pose by a skeleton tree like structure with measured bones lengths. Gait can be captured by a stereovision system of twodimensional video cameras of typical monitoring systems. Such an acquisition stores motion data in the form of video clips - sequences of the two-dimensional images. There is no direct information about the actor positions, skeleton model and its kinematic chain. Motion capture systems, which acquire motion as a time sequence of poses are much more detailed and accurate [4].

We define gait to be the coordinated, cyclic combination of movements that result in human locomotion. The movements are coordinated in the sense that they must occur with a specific temporal pattern for the gait to occur. The movements in a gait repeat as a walker cycles between steps with alternating feet. It is both the coordinated and cyclic nature of the motion that makes gait a unique phenomenon. Examples of motion that are gaits include walking, running, jogging, and climbing stairs. Sitting down, picking up an object, and throwing and object are all coordinated motions, but they are 
not cyclic. Jumping jacks are coordinated and cyclic, but do not result in locomotion [5].

Gait recognition is the process of recognizing many salient properties such as: identity, style of walk, or pathology. This is done based on coordinated and cyclic motions that result in human locomotion [5].

With the increasing demands of visual surveillance systems, human identification at a distance has recently gained more interest. Gait is a potential behavioral feature and many allied studies have demonstrated that it has a rich potential as a biometric for recognition. The development of computer vision techniques has also assured that vision based automatic gait analysis can be gradually achieved. The combination of human motion analysis and biometrics in surveillance systems has become a popular research direction over the past few years. Vision-based human identification at a distance, in particular, has recently gained wider interest from the computer vision community. This interest is strongly driven by the need for automated person identification systems for visual surveillance and monitoring applications in securitysensitive environments such as banks, parking lots, and airports[6][7]. Recently, many researches were focused on gait recognition each with different approaches, advantages and limitations [8..15].

Particle swarm optimization (PSO) is a heuristic, populationbased, self-adaptive search optimization technique that is based on swarm intelligence to solve optimization problems in many applications. It comes from the research on the bird and fish flock movement behavior. The algorithm is widely used and rapidly developed for its easy implementation and few particles required to be tuned [16]. PSO was first introduced in 1995 by Kennedy and Eberhart [17] and has been growing rapidly. Many literature researches were focused on developing and enhancing the PSO [18..26]. PSO was used in many researches for solving recognizing problems such as face recognition [27][28] and palmprint recognition [29..32]. We noted that there is lack of literature researches related to gait recognition that based on PSO. Ivekovic et al. (2008) [33] presented PSO for just upper-body pose estimation. They addressed human body pose estimation from still images. They acquired a multi view set of images of a person sitting at a table is and they estimated pose.

Discrete Cosine Transform (DCT) had been introduced by Ahmed, Natarajan and Rao (1974) [34] and can be regarded as a popular transformation technique widely used in image processing [35][36] and. DCT had been used by many researches [35-41] as a feature extraction in recognition process for dimension reduction.

According to above introduction, PSO is used in this work for gait recognition according to its optimization features. To increase the performance of this suggested gait recognition system, DCT will be used for dimensionality reduction and feature extraction. This paper is organized as follows: section
2 includes description of PSO. Section 3 includes description of DCT. Section 4 includes research methodology and section 5 includes results. Finally section 6 concludes this work.

\section{PRACTICAL SWARM OPTIMIZATION ALGORITHM}

PSO is proposed by Kennedy and Eberhart in 1995 [17]. PSO can be implemented easily, converged rapidly and applied on large number of samples. The PSO includes the following main points [16-26]:

- Each solution is implemented as a particle (N-dimension vector) that represents one individual of a population.

- Each particle has a fitness function (value) associated with it. Each particle adjusts its position and evaluate their position and move closer to optimal point.

- Particles compare themselves to their neighbors and imitate the best of that neighbor.

- Pbest: represents the best value of the particle $i$.

- Gbest: best value that one of the swarm particle reach it.

- Lbest: best value that particle in a local swarm reach it

- Eq.1 used to compute new velocity of each particle: $\mathrm{Vi}(\mathrm{t}+1)=\mathrm{W} \times \mathrm{Vi}(\mathrm{t})+\mathrm{C} 1 \times \operatorname{rand} \times(\operatorname{Pbest}(\mathrm{t})-\mathrm{Xi}(\mathrm{t}))+$

$\mathrm{C} 2 \times \operatorname{rand} \times(\operatorname{Gbest}(\mathrm{t})-\mathrm{Xi}(\mathrm{t}))$

Where, V[]: particle velocity,

$\mathrm{Xi}$ : ith particle of swarm

$\mathrm{W}$ : weight (random number between 0 and 1 ).

$\mathrm{C} 1, \mathrm{C} 2$ : the speeding factors (with value 2 ).

From Eq.1, the new velocity vi $(t+1)$ is affected by: Pbest, Gbest and $\mathrm{Vi}(\mathrm{t})$ : velocity of ith particle $\mathrm{X}$ in time $\mathrm{t}$.

- Eq.2 used to compute new fitness value of each particle: $\mathrm{Xi}(\mathrm{t}+1)=\mathrm{Xi}(\mathrm{t})+\mathrm{Vi}(\mathrm{t}+1)$

The particle will change its value according to its new velocity $(\operatorname{vi}(\mathrm{t}+1))$

PSO algorithm was described in details in researches [16-26]:

1. Initialize parameters (number of generations, population size, weights, c1, c2)

2. Initialize population (velocity and position of each particle) and initialize Pbest and Gbest.

3. New generation

4. Take one particle(P) from population

5. Compute new velocity (Pvelocity) of particle using Eq.1.

6. Compute new position (Pposition) of particle using Eq. 2

7. Pbest $=$ Pposition if $\operatorname{cost}($ Pposition $<=\operatorname{cost}($ Pbest $)$

8. Gbest $=$ Pbest if $\operatorname{cost}($ Pbest $)<=\operatorname{cost}($ Gbest $)$

9. Repeat steps (4..10) until there are more particles in population

10. Repeat steps (3..10) until reaching maximum number of generations

11. Return Gbest 


\section{DISCRETE COSINE TRANSFORM (DCT)}

DCT transforms the input image into a linear combination of weighted basis functions. DCT transform image from spatial domain to frequency domain. DCT uses cosine base functions and exhibits good de correlation and energy compaction characteristics. DCT of an $\mathrm{N} \times \mathrm{M}$ image $\mathrm{f}(\mathrm{x}, \mathrm{y})$ is defined by the following equation [34-36]:

$$
F(u, v)=\alpha(u) \alpha(v) \sum_{x=0}^{N-1} \sum_{y=0}^{M-1} \cdot \cos \left[\frac{\pi \cdot u}{2 \cdot N}(2 x+1)\right] \cos \left[\frac{\pi \cdot u}{2 \cdot M}(2 y+1)\right] f(x, y)
$$

Where $\mathrm{f}(\mathrm{x}, \mathrm{y})$ is the intensity of pixel in row $\mathrm{x}$ and column $\mathrm{y}$, $\mathrm{u}=0,1, \ldots, \mathrm{N}-1$,

$\mathrm{v}=0,1, \ldots, \mathrm{M}-1$,

$\alpha(\mathrm{u}), \alpha(\mathrm{v})$ : functions are defined as following equation [34-36]:

$$
\alpha(u), \alpha(v)= \begin{cases}\sqrt{\frac{1}{N}} & \text { for } u, v=0 \\ \sqrt{\frac{2}{N}} & \text { for } u, v \neq 0\end{cases}
$$

The DCT helps separate the image into parts of differing importance with respect to image's visual quality. For most images, much of signal energy lies at low frequencies. These are relocated to upper-left corner of DCT array. Lower-right values of DCT array represent higher frequencies and turn out to be small to be removed with little visible distortion. The number of DCT coefficients might affect the recognition rate. DCT had been used by many researches as a feature reduction and extraction [34-36].

\section{RESEARCH METHODOLOGY}

The research methodology depends on a database taken from CASIA [42] database with different views that have different silhouette in person's height and width. The Institute of Automation, Chinese Academy of Sciences (CASIA) provide the CASIA Gait Database to gait recognition and related researchers to promote the research. In CASIA Gait Database there are three datasets: Dataset A, Dataset B (multi view dataset) and Dataset $C$ (infrared dataset). Dataset A (former NLPR Gait Database) was created on 10Dec2001 including 20 persons.

Each person has 12 image sequences, 4 sequences for each of three directions (parallel, $45^{\circ}$ and $90^{\circ}$ to image plane). The length of each sequence is not identical for the variation of the walker's speed, but it must ranges from 37 to 127 .

A gait recognition system based on PSO and DCT for feature extraction is suggested in this work. The main steps for PSO for training/testing gait recognition system were implemented using MatLab2013. The DataBase of this system includes 9000 images (each of size $240 \times 352$ pixels) of 15 persons which selected from CASIA database. Each person with 50 images (states) for 4 cases for three angles $(0,45$ and 90). At the end the selected database includes: 15 person $\times 3$ angles $\times 4$ cases $\times 50$ states $=9000$ images.

\section{A. Training Part Of Gait Recognition}

The training part of the gait recognition system is described in Fig. 1 and includes the following steps:

1. Read 50 images (each of size $240 \times 352$ pixels) for each one of the 4 states for each one of the three angles $(0,45$ and 90).

2. The OR logical gate will be applied on each 50 images to produce only one average image for each case of the 4 cases. This is applied for each angle. Then the total number of images resulted from this process are: $15 \times 3 \times 4 \times 1=$ 180 images for 15 persons. Fig. 2 shows the image of person 1 after applying the OR gate on 50 images of gait of person1 for angle $90^{\circ}$. Fig. 3 shows the image of person 1 after applying the OR gate on 50 images of gait of person1 for angle $0^{\circ}$. Whereas Fig.4 shows the image of person 2 after applying the OR gate on 50 images of gait of person 1 for angle $45^{\circ}$.

3. Data standardization. Resize each one of image of size $240 \times 352$ pixels to be image of size $190 \times 100$ pixels. The main goal is producing a dataset with the same position of the person in the middle of each frame and same size in whole image sequence. The idea is to fix the head for each frame in a predefined position and resize the body to achieve a preset height. We perform a three stage preprocessing: extract rectangle including the person without extra black pixels and obtain height and width of the person; sequence is calculated and each frame is converted to biggest height and width; and finally, move head of each frame in a fixed point.

4. Take small block $(70 \times 70,60 \times 60,40 \times 40$ or $20 \times 20)$ from each image of size $190 \times 100$ pixels. We will use different dimension for each experiment to examine which dimension will lead to best recognition rate.

5. Convert each sub image block from two dimensional array to one dimensional array.

6. The person properties will be extracted by applying DCT algorithm for feature extraction.

7. PSO for classification is used for each one of the 180 feature vectors (generated using DCT) as follows: 
Step1: initialize PSO parameters as shown in Table I.

Step 2: initialize position, velocity, Pbest and Gbest.

Step3: Calculate fitness function of each sample

Step4: Calculate optimal value of particle swarm (Pbest) and optimum value of group (Gbest) according to comparison between current value of particle and Pbest and Gbest

Step5: Calculate new speed of practical according to Eq.1. Step6: Compute new position of particle according to Eq.2. Step7: Repeat steps (3-6) while more iterations.

Step8: Store features sub set which are represented by vector with 40 values (according to population size) in sub features database: gaitdbf

\begin{tabular}{|l|l|}
\hline C1 & 2 \\
\hline C2 & 2 \\
\hline Weight & 0.5 \\
\hline Number of Iterations & 100,150 \\
\hline
\end{tabular}

\section{B.Testing Part of Gait Recognition}

The testing part (recognition step) of the suggested system is described in details in Fig.5.

Table I: Parameters of PSO



Fig.1: The Training Part of gait recognition system using PSO and DCT
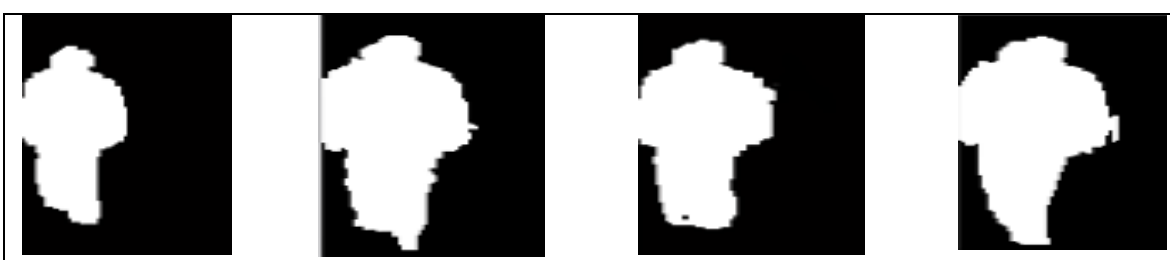

Fig.2: Image of person 1 after applying OR on 50 images for each state of gait of person1 for angle $90^{\circ}$ 
ICIT 2015 The $7^{\text {th }}$ International Conference on Information Technology

doi:10.15849/icit.2015.0023 C ICIT 2015 (http://icit.zuj.edu.jo/ICIT15)

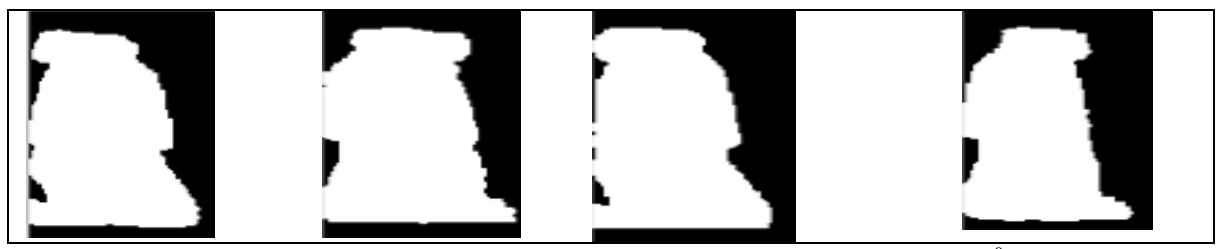

Fig.3: Image of person 1 after applying OR on 50 images of gait of person 1 for angle $0^{0}$

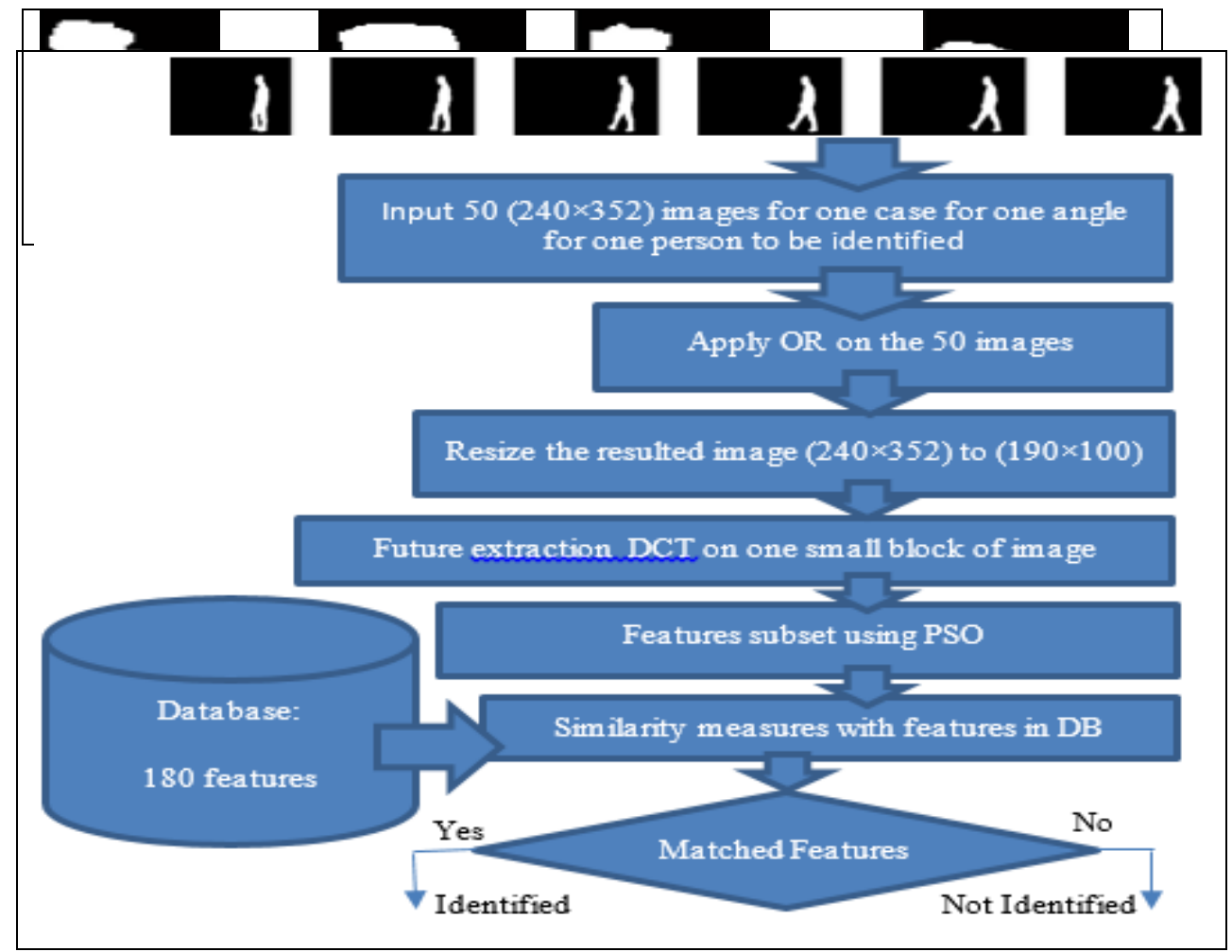

Fig.5: The Testing Part of gait recognition system using PSO and DCT

\section{EXPERIMENTAL RESULTS}

The suggested gait recognition system that is based on PSO and DCT is implemented using MATHLAB 2013. Many experiments were conducted for the suggested gait recognition system. The DataBase of the suggested gait recognition system includes 9000 images (each of size $240 \times 352$ pixels) for 15 persons were selected from CASIA DataBase [42]. Each person with 3 angles $(0,45$ and 90), each angle with 4 cases and 50 images for each case. Each original image is resized from its original dimension to $190 \times 100$ pixels. The performance of the suggested system is computed using recognition ratio, MSE and PSNR.
The first experiment depends on executing the PSO and DCT for feature extraction using various DCT coefficients sizes. The two dimensional DCT is applied to the input image and only a subset of DCT coefficients corresponding to the upper left corner of DCT array is retained.

Different subset sizes of $70 \times 70,60 \times 60,40 \times 40$ and $20 \times 20$ of the original $100 \times 190$ DCT array are used in this experiment as input to the subsequent feature selection phase. 
In this experiment, we determined swarm size equal 40 and 100 iterations. Table II shows the results of using PSO/ DCT with different block size.

TABLE II: PSO/DCT (SWARM=40, ITERATIONS=100)

\begin{tabular}{|l|l|l|l|}
\hline Swarm size N $=40$, No. of Iterations $=100$ \\
\hline subimage & reco.rate & MSE & PSNR \\
\hline $70 \times 70$ & $96 \%$ & 0.0088 & 35 \\
\hline $60 \times 60$ & $92 \%$ & 0.0121 & 32 \\
\hline $40 \times 40$ & $89 \%$ & 0.0187 & 29 \\
\hline $20 \times 20$ & $85 \%$ & 0.0211 & 27 \\
\hline
\end{tabular}

We can note from Table II that best results including high recognition rate and low MSE were obtained when selecting sub image dimension equal $70 \times 70$.

Another experiment was conducted for PSO/DCT with selection of swarm size equal 40 and number of iterations equal 150. At the same time, we determined different sub image dimension for this experiment $(70 \times 70,60 \times 60,40 \times 40$ or $20 \times 20)$. Table III shows results of PSO/DCT with swarm size $\mathrm{N}=40$ and number of iterations equal 150 .

TABLE III: PSO/DCT RESULTS (SWARM=40,ITERATIONS=150)

\begin{tabular}{|l|l|l|l|}
\hline Swarm size N=40 and No. of Iteration $=150$ \\
\hline subsetimage & reco.rate & MSE & PSNR \\
\hline $70 \times 70$ & $95 \%$ & 0.0089 & 34 \\
\hline $60 \times 60$ & $91 \%$ & 0.0134 & 30 \\
\hline $40 \times 40$ & $87 \%$ & 0.0178 & 28 \\
\hline $20 \times 20$ & $84 \%$ & 0.0256 & 26 \\
\hline
\end{tabular}

Other experiments were based on PSO/DCT with swarm size equal 30, number of iterations equal 100 and using different size of sub image dimension $(70 \times 70,60 \times 60,40 \times 40$ or $20 \times 20)$. Table IV shows results of PSO/DCT with swarm size $\mathrm{N}=30$ and number of iterations equal 100.

TABLE IV: PSO/DCT RESULTS (SWARM=30, ITERATIONS=100)

\begin{tabular}{|l|l|l|l|}
\hline Swarm size N=30 AND No. of Iteration $=100$ \\
\hline Subset image & Reco.rate & MSE & PSNR \\
\hline $70 \times 70$ & $95 \%$ & 0.0091 & 34 \\
\hline $60 \times 60$ & $91 \%$ & 0.0125 & 31 \\
\hline $40 \times 40$ & $88 \%$ & 0.0189 & 28 \\
\hline $20 \times 20$ & $84 \%$ & 0.0217 & 27 \\
\hline
\end{tabular}

Other experiments were based on PSO/DCT with swarm size equal 30 , number of iterations equal 150 , with different size of sub image dimension $(70 \times 70,60 \times 60,40 \times 40$ or $20 \times 20)$. Table $\mathrm{V}$ shows results of PSO based LDA/DCT with Swarm size $\mathrm{N}=30$ and number of iteration $=150$

TABLE V: PSO/DCT RESULTS (SWARM=30, ITERATION=150)

\begin{tabular}{|l|l|l|l|}
\hline Swarm size N=30 AND No. of Iteration $=150$ \\
\hline Subset image & Reco.rate & MSE & PSNR \\
\hline $70 \times 70$ & $91 \%$ & 0.0119 & 32 \\
\hline $60 \times 60$ & $87 \%$ & 0.0178 & 28 \\
\hline $40 \times 40$ & $86 \%$ & 0.0220 & 27 \\
\hline $20 \times 20$ & $84 \%$ & 0.0276 & 26 \\
\hline
\end{tabular}

Finally, other experiments were based on PSO/DCT with swarm size equal 20, number of iterations equal 150 , with different size of sub image dimension $(70 \times 70,60 \times 60,40 \times 40$ or $20 \times 20$ ). Table VI shows results of PSO based LDA/DCT with Swarm size $\mathrm{N}=20$ and No. of Iteration $=150$.

TABLE VI: PSO/DCT RESULTS (SWARM=20, ITERATION=150)

\begin{tabular}{|l|l|l|l|}
\hline Swarm size N=20 and No. of Iteration $=150$ \\
\hline Subset image & Reco.rate & MSE & PSNR \\
\hline $70 \times 70$ & $90 \%$ & 0.0122 & 30 \\
\hline $60 \times 60$ & $86 \%$ & 0.0187 & 26 \\
\hline $40 \times 40$ & $84 \%$ & 0.0224 & 25 \\
\hline $20 \times 20$ & $82 \%$ & 0.0287 & 24 \\
\hline
\end{tabular}

From Table III, Table IV, Table V, and Table VI, we can note that the swarm size can affect the overall results (recognition rate, MSE and PSNR. The best results of recognition rate were obtained when selecting swarm size equal 40 with 100 iterations. Also the dimension of the sub image can affect the recognition rate of the system. Best recognition rates for all experiments were obtained when determining sub image dimension equal $70 \times 70$. This is because the big sub image dimension will take more features of the image. Whereas the results related to recognition rate are low when determining sub image dimension equal $20 \times 20$ because small features of image will be taken. Fig. 6 shows that increasing the block size will increase the recognition rate and PSNR of the gait recognition system when selecting swarm size equal 40 and number of iterations equal 100. Fig.7 shows the effect of swarm size on the recognition rate and PSNR when selection bloc dimension equal $70 \times 70$ and 150 number of iterations

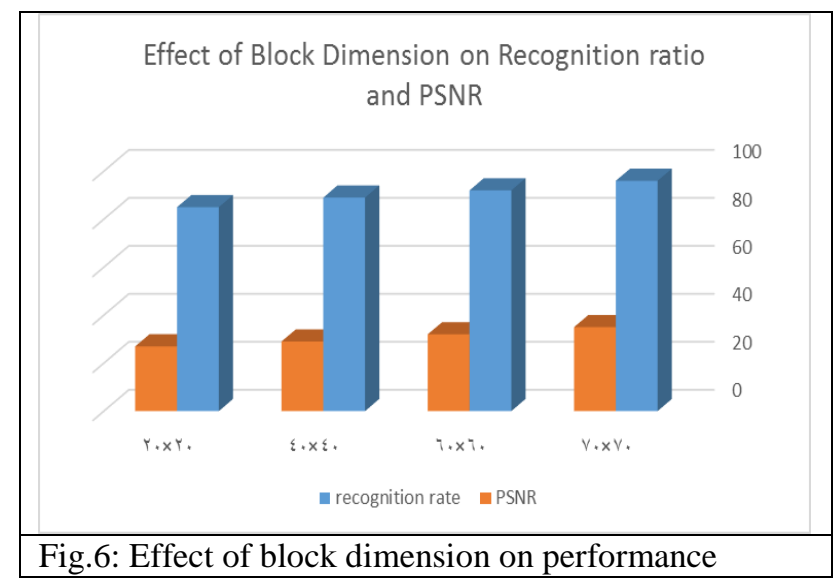

Fig.6: Effect of block dimension on performance 


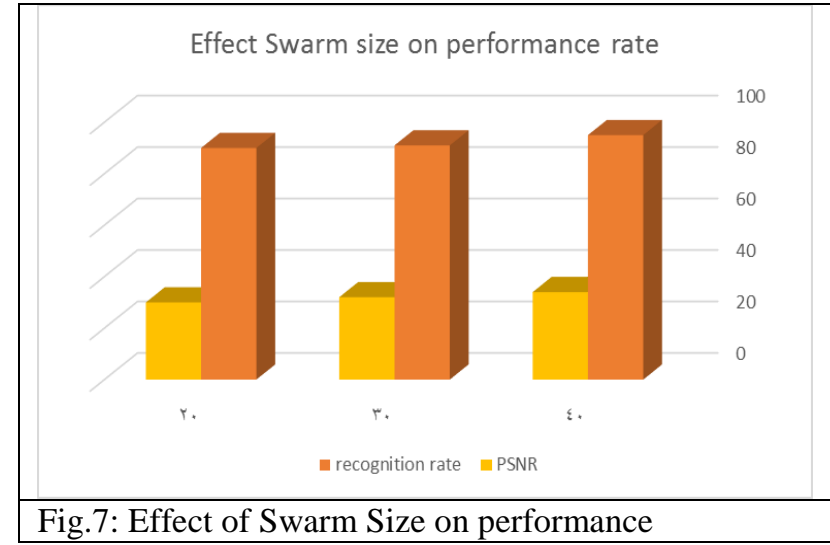

\section{CONCLUSION}

Gait recognition is a type of biometric recognition and related to the behavioral characteristics of biometric recognition. Person identification using gait is method to identify an individual by the way he walk. A gait recognition system was presented in this paper using PSO and DCT for feature reduction and extraction

The gait recognition system was implemented using MatLab 2013. The DataBase of the gait recognition program includes 9000 images (each of size $240 \times 352$ pixels) of 15 persons which selected from CASIA database [42] with different angles (0, 45 and 90), cases (4 cases) and states (50 state for each person. The original images were resized from $240 \times 352$ pixels to $190 \times 100$ pixels.

Many experiments were conducted for executing the gait recognition program based on PSO and DCT with different: swarm size, number of iterations and sub block dimension. The experimental results showed that the best values of recognition rate, MSE and PSNR were obtained when increasing the sub image block size of $70 \times 70$ pixels. Also best results were obtained when increasing the swarm size to 40 . The recognition rate reached $96 \%$, MSE reached 0.0088 and finally PSNR reached $35 \%$.

As a future work, other feature extraction algorithm may be used to reduce the image dimensionality and feature extraction of the image to be used in recognition process. Many experiments will be conducted to make comparisons between different algorithms for feature extraction to determine the suitable algorithm that lead to best recognition performance.

\section{REFERENCES}

[1] Sruthy Sebastian, Activity Based Person Identification Using Particle Swarm Optimization Algorithm, International Journal of Computer Science and Mobile Computing, Vol.2, Issue.7, Jul2013, pp:1-6.

[2] A. K. Jain, A. Ross, S. Prabhakar, "An Introduction to Biometric Recognition", IEEE Trans. on Circuits and Systems for Video Technology, Vol.14, No.1, Jan2004, pp 4-19.
[3] Gajanan P. K., et al. Human Computer Interpreting with Biometric Recognition System, International Journal of Advanced Research in Computer Science and Software Engineering, Vol.2, Issue.12, Dec2012, pp:140-147.

[4] Adam Świtoński, et al. Human Identification Based on the Reduced Kinematic Data of the Gait, 7th International Symposium on Image and Signal Processing and Analysis (ISPA), IEEE, Dubrovnik,4-6 Sep2011, pp:650-655.

[5] M.Tistarelli, J.Bigun, and E.Grosso, Biometric Gait Recognition, Biometrics School 2003, LNCS 3161, 2005, pp: 19-42.

[6] Liang Wang, et al. Silhouette Analysis-Based Gait Recognition for Human Identification, IEEE Transactions On Pattern Analysis And Machine Intelligence, Vol.25, No.12, Dec2003, pp:1-14.

[7] Liang Wang, et al, Fusion of Static and Dynamic Body Biometrics for Gait Recognition, Proceedings of the Ninth IEEE International Conference on Computer Vision (ICCV 2003), Vol.2.

[8] Chiraz BenAbdelkader, et al. EigenGait: Motion-based Recognition of People using Image Self-Similarity, Lecture Notes in Computer Science Vol.2091, 2001, pp:284-294.

[9] Payam S. and Swarup M. and Yuri O., Multi-View Classifier Swarms for Pedestrian Detection and Tracking, IEEE Computer Society Conference on Computer Vision and Pattern Recognition, CVPR Workshops, San Diego, CA, USA, 2525Jun2005.

[10] Qiong C., Bo F., and Hui C., Gait Recognition Based on PCA and LDA, Proceedings of the Second Symposium International Computer Science and Computational Technology(ISCSCT '09), Huangshan, P. R. China, 26-28Dec2009, pp:124-127.

[11] Ra'ul M. and Tao Xiang, Gait Recognition by Ranking, A. Fitzgibbon et al. (Eds.): ECCV 2012, Part I, LNCS 7572, Springer-Verlag Berlin Heidelberg,2012, pp:328-341.

[12] Mohamed Rafi, et al. A Model Based Approach for Gait Recognition System, International Journal of Soft Computing and Engineering (IJSCE) ISSN: 2231-2307, Vol.3, Issue.5, Nov 2013, pp:223-228.

[13] Bogdan Kwolek, et al. 3D Gait Recognition Using SpatioTemporal Motion Descriptors, DOI: 10.1007/978-3-31905458-2_61 In book: Intelligent Information and Database Systems, Publisher: Springer International Publishing, Editors: Nguyen, NgocThanh and Attachoo, Boonwat and Trawiński, Bogdan and Somboonviwat, Kulwadee, 2014, pp.595-604.

[14] Rong Z., Christian V. and Dimitris M., Human Gait Recognition, Conference on Computer Vision and Pattern Recognition Workshop, 27-02Jun2004. CVPRW '04.

[15] Adam S., Andrzej P., Konrad W., Human Identification Based on Gait Paths, book chapter: Advanced Concepts for Intelligent Vision Systems, 13th International Conference, ACIVS 2011, Ghent, Belgium, 22-25 Aug 2011, pp 531-542.

[16] Qinghai Bai, Analysis of Particle Swarm Optimization Algorithm, Computer and Information Science, Vol.3, No.1, 2010.

[17] Kennedy, J. and Eberhart, R., Particle swarm optimization, Proceedings of IEEE International Conference on Neural Networks, Perth, WA, 1995, pp:1942-1948.

[18] Dian P.R., Siti M.S. and Siti S.Y., Particle Swarm Optimization: Technique, System and Challenges, International Journal of Computer Applications (0975 - 8887) Vol.14, No.1, Jan2011, pp:19-27.

[19] Satyobroto Talukder, Mathematical Modelling and Applications of Particle Swarm Optimization, Master's Thesis, Mathematical 
Modelling and Simulation, School of Engineering at Blekinge Institute of Technology, Master of Science, Feb 2011.

[20] Voratas K., Comparison of Three Evolutionary Algorithms: GA, PSO, and DE, Industrial Engineering \& Management Systems, Vol.11, No.3, Sep2012, pp:215-223.

[21] H. Kuo, J. Chang and C. Liu, Particle Swarm Optimization For Global Optimization Problems, Journal of Marine Science and Technology, Vol. 14, No. 3, 2006, pp:170-181.

[22] Daniel Bratton and James Kennedy, Defining a Standard for Particle Swarm Optimization, Proceedings of the 2007 IEEE Swarm Intelligence Symposium, SIS, 2007.

[23] Davoud S. and Ellips M., Particle Swarm Optimization Methods, Taxonomy and Applications, International Journal of Computer Theory and Engineering, Vol. 1, No. 5, Dec 2009, pp: 486-502.

[24] M. Peyvandi, M. Zafarani and E. Nasr, Comparison of Particle Swarm Optimization and the Genetic Algorithm in the Improvement of Power System Stability by an SSSC-based Controller, Journal of Electrical Engineering \& Technology Vol. 6, No. 2, , 2011, pp:182-191.

[25] Bijayalaxmi Panda, Soumya Sahoo, Sovan Kumar Patnaik, A Comparative Study of Hard and Soft Clustering Using Swarm Optimization, International Journal of Scientific \& Engineering Research, Vol.4, Issue.10, Oct2013, pp:785-790.

[26] Rania Hassan, Babak Cohanim, Olivier de Weck, A Copmarison Of Particle Swarm Optimization And The Genetic Algorithm, American Institute of Aeronautics and Astronautics, 46th Aiaa/Asme/Asce/Ahs/Asc Structures, Structural Dynamics And Materials Conference, 2005.

[27] R. M. Ramadan and R. F. AbdelKader, Face Recognition Using Particle Swarm Optimization-Based Selected Features, International Journal of Signal Processing, Image Processing and Pattern Recognition, Vol.2, No.2, Jun2009, pp:51-66.

[28] P.V. Shinde, B.L. Gunjal and R. G. Ghule, Face Recognition Using Particle Swarm Optimization, Emerging Trends in Computer Science and Information Technology 2012(ETCSIT2012) Proceedings published in International Journal of Computer Applications (IJCA), pp:11-13.

[29] M. Arunkumar And S. Valarmathy, Palmprint And Face Based Multimodal Recognition Using Pso Dependent Feature Level Fusion, Journal Of Theoretical And Applied Information Technology,Vol.57, No.3, 30November 2013, pp:337-346.

[30] K. Krishneswari And S. Arumugam, Intra Modal Feature Fusion Based On PSO For Palmprint Authentication, ICTACT Journal On Image And Video Processing, May 2012, Vol.2, Issue.4, pp:435-440.

[31] Ola M. Aly, et al., A Multimodal Biometric Recognition system using feature fusion based on PSO, International Journal of
Advanced Research in Computer and Communication Engineering, Vol. 2, Issue.11, Nov2013, pp: 4336-4343.

[32] Ola M. Aly, et al., An Adaptive Multimodal Biometrics System using PSO, International Journal of Advanced Computer Science and Applications, Vol. 4, No.7, 2013, pp:158-165.

[33] S. Ivekovic, E. Trucco and Y. R. Petillot, Human Body Pose Estimation With Particle Swarm Optimisation, Evolutionary Computation, Vol.16, No.4, pp: 509-528.

[34] M. Ahmad, T. Natarajan and K. R. Rao, Discrete Cosine Transform, IEEE Trans. Computers, 1974, pp:90-94.

[35] Aman R. C., Pallavi P. V. and M. M. Roja, Face Recognition Using Discrete Cosine Transform for Global and Local Features, Proceedings of the 2011 International Conference on Recent Advancements in Electrical, Electronics and Control Engineering (ICONRAEECE), IEEE Xplore., 2011.

[36] Ziad M. Hafed and Martin D. Levine, Face Recognition Using the Discrete Cosine Transform, International Journal of Computer Vision, Vol.43, No.3, 2001 Kluwer Academic Publishers. Manufactured in The Netherlands, 2001, pp:167188.

[37] C. Podilchuk and X. Zhang, "Face Recognition Using DCTBased Feature Vectors," Proc. IEEE International Conference on Acoustics, Speech and Signal Processing (ICASSP'96), vol. 4, May1996, pp. 2144-2147.

[38] Z. Yankun and L. Chongqing, "Efficient Face Recognition Method based on DCT and LDA," Journal of Systems Engineering and Electronics, Vol. 15, No. 2, pp:211-216, 2004.

[39] F. M. Matos, L. V. Batista, and J. Poel, "Face Recognition Using DCT Coefficients Selection," Proc. of the 2008 ACM Symposium on Applied Computing, (SAC'08), March 2008, pp:1753-1757.

[40] Z. Pan and H. Bolouri, "High Speed Face Recognition Based on Discrete Cosine Transform and Neural Networks," Technical Report, Science and Technology Research Center (STRC), University of Hertfordshire.

[41] Virendra P. Vishwakarma, Sujata Pandey Member IEEE, and M. N. Gupta, A Novel Approach for Face Recognition Using DCT Coefficients Re-scaling for Illumination Normalization, 15th International Conference on Advanced Computing and Communications, IEEE Computer Sociaety, pp:535-539.

[42] CASIA Gait Database, http:// www.sinobiometrics.com, 2006. CASIA Gait Database collected by Institute of Automation, Chinese Academy of Sciences" and a citation to "CASIA Gait Database, http://www.sinobiometrics.com" should be added into the references, 2006. 